\section{Journal of Theoretical Biology}

www.elsevier.com/locate/jtbi

\title{
An error catastrophe in cancer?
}

\author{
Ricard V. Soléc ${ }^{\mathrm{a}, \mathrm{b}, *}$, Thomas S. Deisboeck ${ }^{\mathrm{c}, \mathrm{d}}$ \\ ${ }^{a}$ ICREA-Complex Systems Lab, Universitat Pompeu Fabra, Dr Aiguader 80, 08003 Barcelona, Spain \\ ${ }^{\mathrm{b}}$ Santa Fe Institute, 1399 Hyde Park Road, NM 87501, USA \\ ${ }^{\mathrm{c}}$ Complex Biosystems Modeling Laboratory, Harvard-MIT (HST) Athinoula A. Martinos Center for Biomedical Imaging, HST-Biomedical \\ Engineering Center, Massachusetts Institute of Technology, Cambridge, MA 02139, USA \\ ${ }^{\mathrm{d}}$ Molecular Neuro-Oncology Laboratory, Massachusetts General Hospital, Harvard Medical School, Boston, MA 02129, USA
}

Received 26 February 2003; received in revised form 20 August 2003; accepted 26 August 2003

\begin{abstract}
A comparison between the evolution of cancer cell populations and RNA viruses reveals a number of remarkable similarities. Both display high levels of plasticity and adaptability as a consequence of high degrees of genetic variation. It has been suggested that, as it occurs with RNA viruses, there is a threshold in the levels of genetic instability affordable by cancer cells in order to be able to overcome selection barriers (Trends Genet. 15 (1999) M57). Here we explore this concept by means of a simple mathematical model. It is shown that an error threshold exists in this model, which investigates both competition between cancer cell populations and its impact on overall tumor growth dynamics. Once the threshold is reached, the highly unstable tumor cell populations, which were sustaining malignant growth, become unable to maintain their genetic information, which in turn triggers a slowed down overall tumor growth regime.
\end{abstract}

(C) 2003 Elsevier Ltd. All rights reserved.

Keywords: Cancer; RNA viruses; Quasispecies; Genomic instability; Genetic instability; Tumor progression; Phase transitions; Error threshold

\section{Introduction}

A hallmark of cancer cells is their underlying genetic instability. Often, this term is used to describe a state, i.e., the occurrence of either small genetic alterations such as nucleotide deletions or insertions or larger ones such as alterations in the chromosomal number per se, termed aneuploidy. Nonetheless, as Lengauer et al. (1998) already pointed out, genetic instability should denote a rate more than a state, hence the occurrence of a particular mutation in the genome of the cell over time. Such hypermutability has been liked to chemical carcinogens (Bardelli et al., 2001) as well as non-DNA damaging stress ( $\mathrm{Li}$ et al., 2001). Different aspects of hypermutation and its impact on cancer development have been analysed from mathematical models (Wodarz and Krakauer, 2001; Frank et al., 2003; Gatenby and Frieden, 2002; Plotkin and Nowak, 2002).

*Corresponding author. ICREA-Complex Systems Lab, Universitat Pompeu Fabra, Dr Aiguader 80, 08003 Barcelona, Spain. Tel.: + 3493-5422821; fax: +34-93-2213237.

E-mail address: ricard.sole@cexs.upf.es (R.V. Solé).
Given the common conception of tumorigenesis as a multistep process (Fearon and Vogelstein, 1990), with continually accumulating mutations, and based on the calculation that the normal, somatic mutation rate accounts for only $1.4 \times 10^{-10}$ mutations/base pair/ cell generation, Loeb (1991) postulated the existence of a so-called mutator phenotype. As the cited background mutation rate cannot account for the marked heterogeneity seen in most solid cancers, he further argues that "cancer cells must exhibit or have exhibited a mutator phenotype" (Loeb, 1994). Loeb concludes that this mutator hypothesis as well as Novell's generally accepted concept of clonal evolution as the driving force for tumor progression are not exclusive (Novell, 1976). Others, however, have argued that selection without an increased mutation rate is both necessary and sufficient to explain tumorigenesis (Tomlinson et al., 1996) and to account for the mutations seen in tumors, especially if the process of aging were to increase the selective conditions for clonal expansion (Chow and Rubin, 2000).

As discussed by Cahill et al., genetic instability in cancer allows to overcome selection barriers, a process 
which fosters further tumor progression (Cahill et al., 1999; see also Jackson and Loeb, 1998). Normal cells display low levels of instability and tumor progression will benefit from genetic instability by generating cellular diversity. Such heterogeneous populations will include genetic alterations to overcome the barriers and tumor progression will continue (see for example Wodarz and Krakauer, 2001). Although this situation is known to play a key role in the initial stages of tumorigenesis, genetic instability will be observable at late stages: it is carried along with the clonally selected alterations.

A relevant point is the fact that too high levels of instability might be harmful because of accumulated damage. In other words, a limit to instability must exist (Cahill et al., 1999). Such a threshold is actually very similar to the so-called error catastrophe (or error threshold) displayed by RNA viruses (Domingo and Holland, 1994; Domingo et al., 1995; Nowak and May, 2000). These viruses are known to mutate at very high rates. As predicted by Eigen and Schuster's theory of quasispecies, a critical mutation rate exists beyond the genomic information is lost i.e. no Darwinian selection operates (Eigen, 1971; Schuster, 1994). This actually corresponds to an example of a phase transition in a complex biosystem and as such it allows to develop powerful theoretical approximations (Solé et al., 1996; Solé and Goodwin, 2001).

RNA viruses are known to replicate close to their error threshold. Several theoretical approaches to this problem have been developed in order to understand the presence and implications of this threshold (Swetina and Schuster, 1982; Eigen et al., 1987, 1988; Pastor-Satorras and Solé, 2001; Kamp and Bornholdt, 2002; Kamp et al., 2003). The evolutionary success of RNA viruses is due to their enormous plasticity and adaptability to changing environments. The high mutation rate generates a highly heterogeneous population, so-called molecular quasispecies. The quasispecies structure provides an extraordinary reservoir of variants with potentially useful phenotypes in the face of environmental change.

How strong are the similarities between unstable tumors and RNA populations? Several features are clearly shared by both, at least qualitatively. One is the presence of high levels of heterogeneity, both at the genotype and phenotype levels. Different replication and infection mechanisms in RNA viruses are matched by wide levels of variability in cancer cells, affecting cell communication, growth and apoptosis. Accordingly, escape from the immune system (and other selection barriers) operates in both systems. Viruses use antigenic diversity whereas tumors evade the immune system by loosing their antigens through mutation, or making use of antigenic modulation and/or tumor-induced immune suppression (Rosenberg, 2001).

Increased mutagenesis beyond the error catastrophe can destroy the virus, since beyond the threshold no
Darwinian selection is at work (Schuster, 1994). The exceptionally high mutation rates in RNA viruses is illustrated by the finding that most HIV virions in blood appear to be non-viable (Coffin, 1995). Similarly, genetic instability in cancer cells will have detrimental effects on cell's fitness, since most random mutations are likely to be harmful (see Gatenby and Frieden, 2002). Effective experimental strategies have shown that the error threshold can actually be exploited in antiviral therapy (Holland et al., 1999; Loeb et al., 1999; Cottry et al., 2001). Within the context of HIV treatment, using promutagenic nucleoside analogs, viral replication of HIV has been shown to be abolished in vitro (Loeb et al., 1999). As indicated by Cahill et al., the best chance of cure advanced cancers might be a result of tumor genetic instability (Cahill et al., 1999; Loeb et al., 2003): cancer cells are more sensitive to stress-inducing agents. Cancer, it is argued, would provide a good target for direct attack by drugs promoting genetic instability selectively in tumor cells.

To elucidate the possible implications of the error threshold in cancer from a theoretical standpoint, we present a simple mathematical model, which investigates the interplay between cell proliferation and mutation rate within a simple tumor growth model. The results support the notion of the existence of an error-threshold in tumor systems, which corresponds to a "critical" value of genetic instability, which, once exceeded, will trigger a concomitant sharp decrease in the tumor's proliferation rate. This concept of a trade-off between two of the tumor's key features therefore combines for the first time the still controversial "mutator phenotype" hypothesis with the generally accepted, clonal selectiondriven optimization process. Important implications of this work for both experimental studies and future clinical work are discussed.

\section{The error threshold in quasispecies}

In this section we shortly review the simplest model of quasispecies dynamics, the so-called Swetina-Schuster model (1982). The starting point is the general EigenSchuster quasispecies model, defined by the following set of equations:

$\frac{\mathrm{d} x_{i}}{\mathrm{~d} t}=\sum_{j=1}^{n} x_{j} f_{j} Q_{j i}-\Phi(\mathbf{x}) x_{i}$,

where $x_{i}$ indicates the fraction of the population associated to the $i$-th mutant genome (here $i=1, \ldots, n$, where $n$ is very large) so that $\sum_{j=1}^{n} x_{j}=1$. Here $f_{j}$ is the growth rate of the $j$-th mutant, $Q_{i j}$ is the probability of having a mutation $i \rightarrow j$ and $\Phi(\mathbf{x})$ is the average fitness associated to the population vector $\mathbf{x}=x_{1}, \ldots, x_{n}$, i.e., $\Phi(\mathbf{x})=\sum_{j=1}^{n} f_{j} x_{j}=\langle f\rangle$. 
In its simplest form, we can consider a reduced system of equations defining a population as formed by two basic groups: the master sequence $x_{1}$ and the other sequences, which we assume to be grouped into an "average" sequence with population $x_{2}$ (Swetina and Schuster, 1982). Let us also assume (as a first approximation) that mutations occur from the master to the second compartment but not in the reverse sense. The enormous size of the sequence space makes this assumption a good first approximation:

$\frac{\mathrm{d} x_{1}}{\mathrm{~d} t}=f_{1} x_{1} Q-x_{1} \Phi\left(x_{1}, x_{2}\right)$,

$\frac{\mathrm{d} x_{2}}{\mathrm{~d} t}=f_{1} x_{1}(1-Q)+f_{2} x_{2}-x_{2} \Phi\left(x_{1}, x_{2}\right)$,

where it is assumed that $f_{1}>f_{2}$, i.e. the master sequence replicates faster than the mutant sequences. This oversimplified model allows us to see the error threshold condition under a mean field argument.

The fixed points here are located on the line $x_{1}^{*}=$ $1-x_{2}^{*}$, with

$x_{2}^{*}=\frac{f_{1}(1-Q)}{f_{1}-f_{2}}$.

The equilibrium of the previous points is obtained from the Jacobi matrix

$\mathbf{L}_{\mu}\left(x^{*}\right)=\left(\begin{array}{cc}f_{1} Q-\Phi(\mathbf{x})-x_{1} \frac{\partial \Phi}{\partial x_{1}} & -x_{1} f_{2} \\ f_{0}(1-Q)-x_{2} f_{1} & f_{2}-\Phi(\mathbf{x})-x_{2} \frac{\partial \Phi}{\partial x_{2}}\end{array}\right)$.

It can be shown, by means of standard stability analysis that the state where the master sequence gets extinct (i.e. $\left.\left(x_{1}^{*}, x_{2}^{*}\right)=(0,1)\right)$ provided that the eigenvalues of the matrix

$\mathbf{L}_{\mu}(0,1)=\left(\begin{array}{cc}f_{1} Q-f_{2} & 0 \\ f_{0}(1-Q) & -f_{2}\end{array}\right)$ are negative. This leads to

$f_{1} Q<f_{2}$

otherwise, the master sequence is able to survive and Darwinian selection keeps operating. Once the mutation rate exceeds this error threshold, no stable master sequence can persist. The critical condition $Q_{c}=f_{2} / f_{1}$ is shown in Fig. 1(a), separating the quasispecies domain from the drifting phase. The equilibrium population of the master sequence $x_{1}^{*}$ is shown in Fig. 1(b) against the mutation rate $1-Q$. A linear decay is observed until the threshold $Q_{c}$ is reached.

In the following section, the previous model will be extended to a different situation: the growth of a tumor involving genetic instability. As will be shown, although different in a number of ways, the process of tumorigenesis under genetic instability can be mapped into a quasispecies model displaying the error catastrophe.

\section{The error threshold in cancer}

In neoplastic cells, genetic alterations can arise from a disparate source of mechanisms, e.g. from inaccurate DNA replication to failure of DNA repair systems. And indeed, the latter has been recently discussed in the context of a biological equivalent to the mutator phenotype as Fishel (2001) linked the selection for mismatch repair defect in hereditary non-polyposis colon cancer to resistance to DNA damage-induced apoptosis. Previous models of cancer growth and evolution have considered competitive interactions among different cellular populations, usually tumor and host cells (Gatenby, 1995, 1996). Here we extend this type of approach by incorporating mutations between different populations. In a simplified setting, we consider a tumor cell population exhibiting genetic instability as composed by three cell subpopulations,
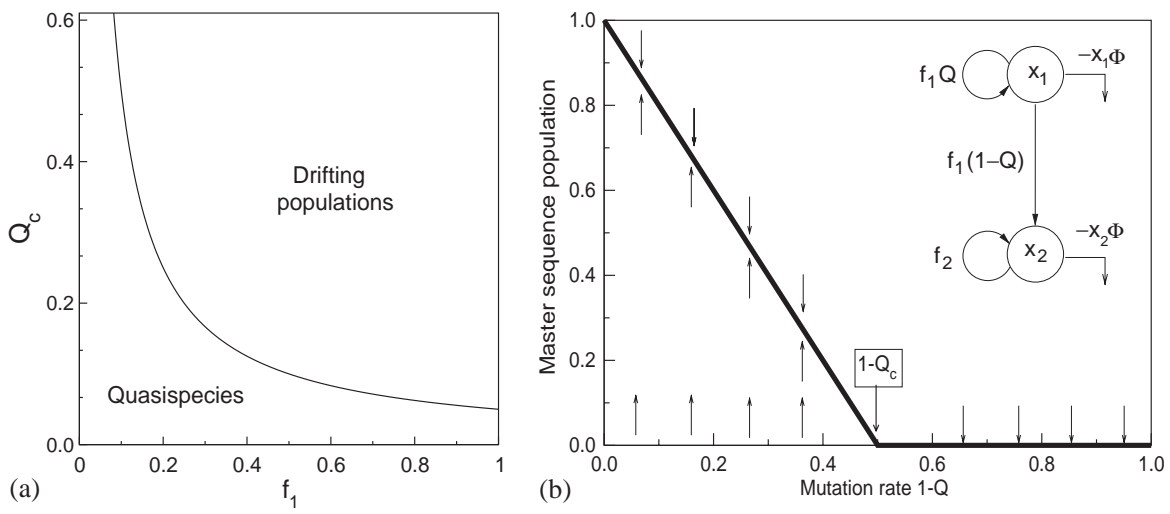

Fig. 1. The basic model of quasispecies dynamics involving only two populations of replicons: the master sequence $\left(x_{1}\right)$ and the other sequences $\left(x_{2}\right)$ that define the population (inset). Mutations occur at a rate $\mu=1-Q$. Here $Q$ is the probability of replication without errors. In (a) the critical curve (for $f_{2}=0.05$ ) $Q_{c}=f 2 / f 1$ is shown against the replication rate of the master sequence. In (b) The large diagram shows an example of the theoretical prediction of the Swetina-Schuster mean field model (see text). Here we have $f_{1}=0.5, f_{2}=0.25$ and thus $Q_{c}=0.5$. 


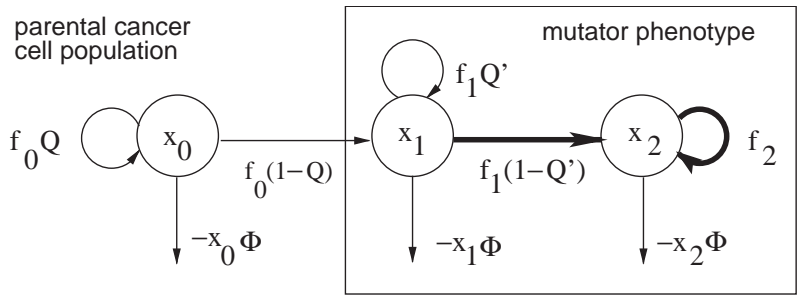

Fig. 2. Mean-field model of a cancer cell population including three basic compartments: a cell population with anomalous growth but no genetic instability $x_{0}$, a population derived from it involving a mutation that allows for genetic instability $\left(x_{1}\right)$ and a third one that is composed by the set of all mutant sequences $x_{2}$.

which correspond each to an increasing number of mutations, hence representing a raising level of genetic instability (Fig. 2). The first one exhibits cancer growth but is generally considered genetically stable, the second, advanced one exhibits genetic instability (with a mutation in a stability controlling gene, involved in generating a mutator phenotype) and a third, heterogeneous population of mutants derived from the second.

The mean-field model here would be obtained from the following system:

$$
\begin{aligned}
\frac{\mathrm{d} x_{0}}{\mathrm{~d} t}= & f_{0} x_{0} Q-x_{0} \Phi\left(x_{0}, x_{1}, x_{2}\right), \\
\frac{\mathrm{d} x_{1}}{\mathrm{~d} t}= & f_{1} x_{1} Q^{\prime}+f_{0} x_{0}(1-Q)-x_{1} \Phi\left(x_{0}, x_{1}, x_{2}\right), \\
\frac{\mathrm{d} x_{2}^{i}}{\mathrm{~d} t}= & f_{1}\left(1-Q_{i 1}^{\prime}\right) x_{1}+f_{2}^{i} \mu_{i i} x_{2}^{i} \\
& +\sum_{j} f_{j} \mu_{i j} x_{2}^{j}-x_{2} \Phi\left(x_{0}, x_{1}, x_{2}\right) .
\end{aligned}
$$

In this model, the most unstable population is indicated by a set of subpopulations $\left\{x_{2}^{i}\right\}$ with $i=1, \ldots, n$. Here some of them will result from mutations in $x_{1}$, which will occur at rates $1-Q_{i 1}^{\prime}$. Additionally, cross-mutations connect the different sub-clones through the mutation term $\sum_{j} f_{j} \mu_{i j} x_{2}^{j}$ where $\mu_{i j}$ indicate the mutation rates from $x_{2}^{j}$ to $x_{2}^{i}$. Following the approach used in the previous section, we can lump together the previous set into a single, average population $x_{2}=\sum_{i} x_{2}^{i}$. Now the third equation would read:

$\frac{\mathrm{d} x_{2}}{\mathrm{~d} t}=f_{1} x_{1}\left(1-Q^{\prime}\right)+f_{2} x_{2}-x_{2} \Phi\left(x_{0}, x_{1}, x_{2}\right)$.
In this simplified system, two different mutation rates are involved, $\mu_{0}=1-Q$ and $\mu_{1}=1-Q^{\prime}$. The first is simply the rate at which cells with mutator phenotype emerge from the non-unstable population (which is here considered as an homogeneous compartment). The second defines the degree of instability displayed by this population, and it lumps together an extremely heterogeneous set of sequences, with some average rate $f_{2}$ that we should assume to be some function of $Q^{\prime}$, since very large instability rates should be largely deleterious. Although the model does not explicitly consider environmental constraints, such as blood supply, hypoxia or acidosis, they can be considered as implicitly introduced through the $\left\{f_{i}\right\}$ set.

For $Q^{\prime}=1$ the previous model reduces to the initial two-dimensional quasispecies model. A new ingredient needs to be introduced here: the deleterious effects of high genetic instability on the fitness of the unstable population $x_{2}$. This can be easily included by considering a functional relation

$f_{2}=\alpha f_{1} \phi\left(\mu_{1}\right)$,

where $\phi\left(\mu_{1}\right)$ will be a decreasing function such that $\phi(0)=1$, indicating the speed of fitness decays as mutation increases and $\alpha>1$ is a competitive advantage parameter, indicating how much faster the $x_{2}$ population grows in relation with the unstable phenotype $x_{1}$ population. For $\alpha=1$ we would have the same replication rates and only two (effective) cell types.

Three different scenarios are considered here in relation with the functional response $\phi\left(\mu_{1}\right)$ : (a) linear decay, which assumes that $\phi\left(\mu_{1}\right)=1-\theta \mu_{1}$ (here $\theta=5$ ). A more realistic scenario involves (b) an exponential decay with mutation rate, i.e. $\phi\left(\mu_{1}\right)=\exp \left(-\theta \mu_{1}\right)$ (here $\theta=5$ ). A third situation would involve a threshold function: $\phi\left(\mu_{1}\right)=1-\mu_{1}^{z} /\left(\mu_{1}^{z}+\theta_{1}^{z}\right)$ with $\theta \in[0,1]$ and $z>1$ (here $z=5$ and $\theta=0.2$ ). The parameter $\theta$ would play the role of a threshold mutation rate where a sharp decrease in fitness would take place, whereas for $\mu_{1}<\theta$ the fitness remains constant.

The previous system displays two attractors, one involving the dominance of the unstable population (i.e. $\left.\mathbf{x}_{u}^{*}=(0,0,1)\right)$ corresponding to malignant, fast growth and another where the three clones coexist. The stability of these two attractors is performed by using the Jacobi matrix, which here reads as

$$
\mathbf{L}_{\mu}\left(x^{*}\right)=\left(\begin{array}{ccc}
f_{0} Q-\Phi(\mathbf{x})-x_{0} \frac{\partial \Phi}{\partial x_{0}} & -x_{0} \frac{\partial \Phi}{\partial x_{1}} & -x_{0} \frac{\partial \Phi}{\partial x_{2}} \\
f_{0}(1-Q)-x_{1} \frac{\partial \Phi}{\partial x_{0}} & f_{1} Q^{\prime}-\Phi(\mathbf{x})-x_{1} \frac{\partial \Phi}{\partial x_{1}} & -x_{1} \frac{\partial \Phi}{\partial x_{2}} \\
-x_{2} \frac{\partial \Phi}{\partial x_{0}} & f_{1}\left(1-Q^{\prime}\right)-x_{2} \frac{\partial \Phi}{\partial x_{1}} & f_{2}-\Phi(\mathbf{x})-x_{2} \frac{\partial \Phi}{\partial x_{2}}
\end{array}\right)
$$


Here, given the definition of $\Phi(\mathbf{x})$, we have $\partial \Phi / \partial x_{i}=f_{i}$.

We are here interested in the domain at which the malignant growth becomes dominant and its stability. The stability analysis of the fixed point $\mathbf{x}_{u}^{*}=(0,0,1)$ leads to the following eigenvalues from the Jacobi matrix:

$\lambda_{1}=f_{0} Q-f_{2}, \quad \lambda_{2}=f_{1} Q^{\prime}-f_{2}, \quad \lambda_{3}=-f_{2}$,

which imposes the two inequalities $f_{0} Q<f_{2}$ and $f_{1} Q^{\prime}<f_{2}$ to be satisfied simultaneously. Using the mutation rates $\mu_{0}=1-Q$ and $\mu_{1}=1-Q^{\prime}$ the stability of $\mathbf{x}_{u}^{*}$ is satisfied provided that

$\mu_{0}<\frac{f_{2}-f_{0}}{f_{0}}, \quad \mu_{1}<\frac{f_{2}-f_{1}}{f_{1}}$.

Since $\mu_{0} \ll \mu_{1}$, the first inequality is expected to be satisfied and thus we can concentrate in the second one. This can be re-written as

$f_{1}\left(\mu_{1}+1\right)<f_{2}\left(\mu_{1}\right)$

and using the previous assumption for the functional response, we have the stability condition

$\alpha>\alpha_{c}=\frac{\mu_{1}+1}{\phi\left(\mu_{1}\right)}$.

In Fig. 3 the phase space for these three different situations is shown, where the domain of genetic instability is indicated in gray shade. The line separating the two domains describes an error-threshold condition: as we move from left to right starting from a point in the gray zone, we cross from a domain of malignant growth to a domain (sharply separated from the first) where growth takes place at the rate defined by $f_{0}$ (slow growth). An example of the changes experienced by the three populations is shown in Fig. 3 (lower set). Here the continuous, dashed and thick lines correspond to the $x_{0}, x_{1}$ and $x_{2}$-populations, respectively.

The sharp character of this transition is better appreciated in Fig. 4, where the stationary population of the mutators $x_{2}$ is shown against $\alpha$ and $\mu_{1}$. The plateau corresponds to the malignant growth phase, where $x_{2}$ dominates. The boundary defining the error catastrophe is very well defined and the rapid decline clear. This decay is actually sharper for higher values of $\alpha$. In other words, the faster replicating the unstable population, the easier to drop to minimum levels. This indicates that genetic instability behaves in a rather allor-none way suggesting that increased levels of mutation would stop malignant growth. This observation fits the qualitative prediction suggesting that genetic instability is thresholded so that appropriate levels of instability allow to overcome selection barriers but too much instability leads to extinction of the unstable clone (Cahill et al., 1999).
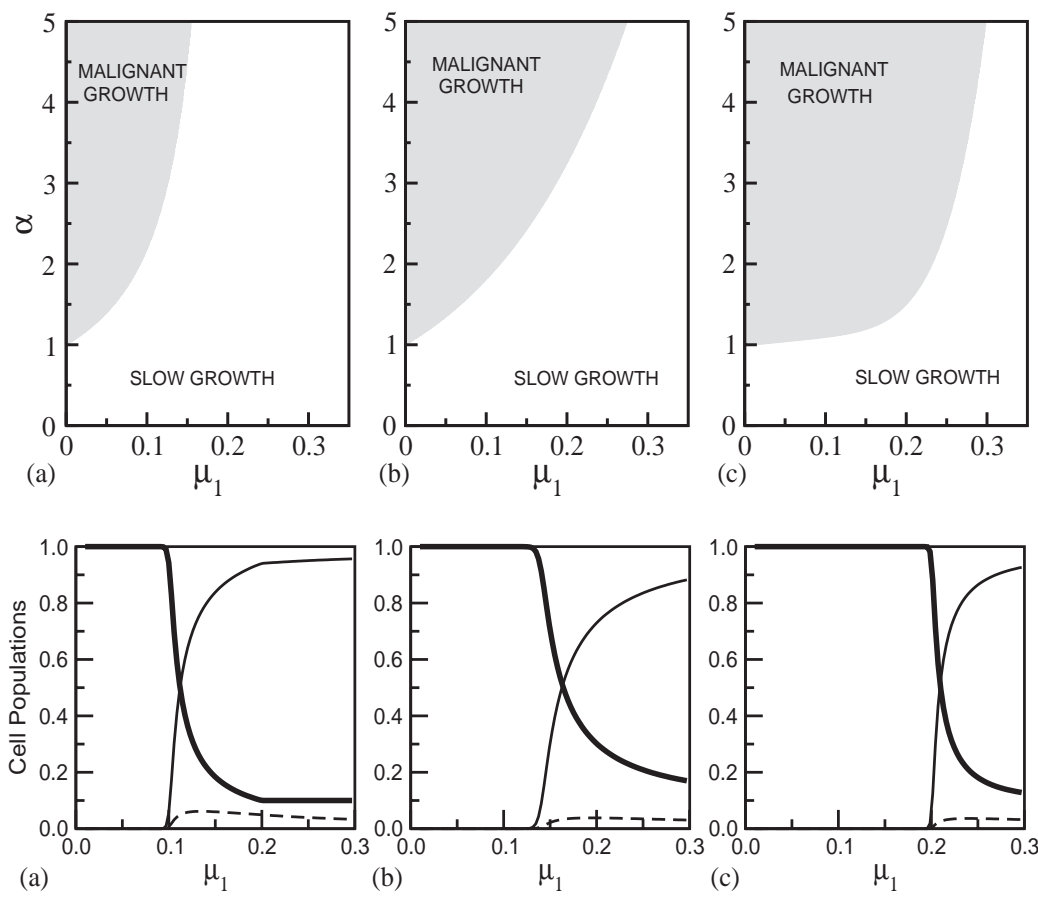

Fig. 3. Phase space of the cancer quasispecies model. Here the competitive advantage $\alpha$ is shown against the mutation rate $\mu_{1}$. The upper domain defines the parameter space where malignant growth takes place, while the lower domain involves slow-growing tumor cell clones. Three scenarios are considered: (a) linear, (b) exponential and (3) threshold (see text). The lower pictures show an example of the equilibrium populations reached for $\alpha=2$ at different mutation rates. 


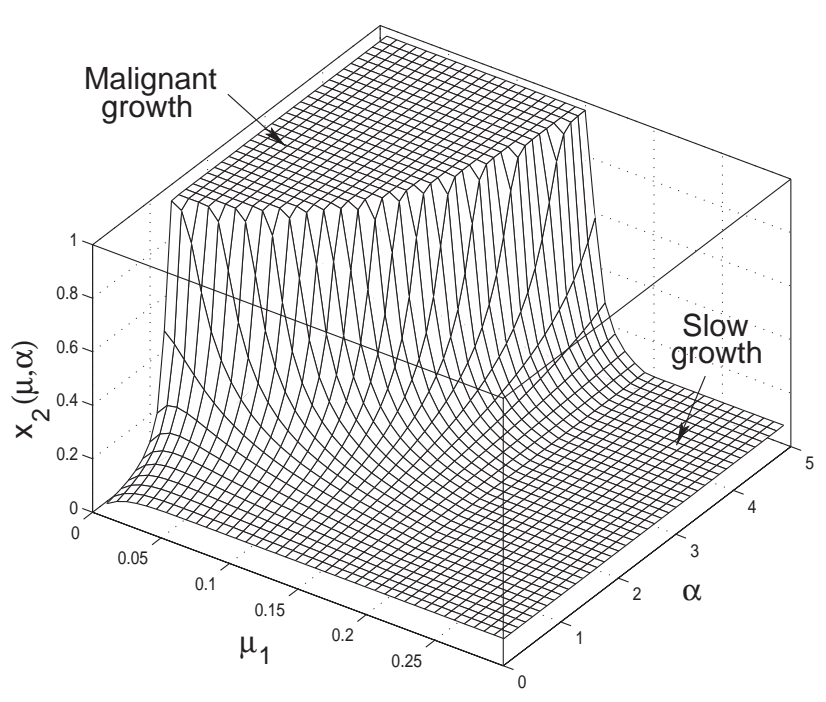

Fig. 4. The equilibrium population of the unstable set $x_{2}$ as a function of $\mu_{1}$ and $\alpha$ for the linear case $\phi\left(\mu_{1}\right)=1-\theta \mu_{1}$ (here $\theta=5$ ). We can clearly appreciate the sharp decay close to the boundaries defined by the critical condition $\alpha=\left(\mu_{1}+1\right) / \phi\left(\mu_{1}\right)$.

\section{Discussion}

In recent years, it has become increasingly clear that many diseases cannot be treated without taking into account their multifactorial character. This recognition led to the coining of a new term, complex disease to label this family of illnesses where many different factors or genes are involved. Cancer belongs to this family of complex diseases, thus needs to be investigated as such and targeted accordingly. Based on PCR analysis of samples from colorectal premalignant polyp and carcinoma cell genomes estimated that about 11000 genomic alterations occur in a cancer cell. Such instability provides ample opportunities for the emergence of the clonal heterogeneity seen in many human cancers. This is of clinical importance as tumor cell subpopulations have been shown to interact with each other, thereby affecting their growth rate, chemo-sensitivity and metastatic phenotype (e.g. Miller et al., 1981, 1987; Poste et al., 1981).

Over the years, one of the more controversial questions in cancer research has been if the vast amount of mutations found in human tumors can be the product of the normal, somatic mutation rate only, guided by selection pressures or if it requires some sort of a hypermutability, termed a mutator phenotype population and if so, if both seemingly mutually exclusive concepts could possibly be combined to one paradigm. Theoretical models such as the one presented here can help address this question, thus possibly yield new, important insights into the complexity of tumor progression, which in turn may lead to novel and experimentally testable hypotheses and, eventually, more successful clinical approaches.
Based on the underlying assumption of the existence of a mutator-phenotype population, our results support the notion that such an error threshold is operating in cancer as well. Specifically, if the level of genetic instability conferred through the mutation rate $\mu_{1}$, of the mutator-phenotype population, $x_{1}$, exceeds a certain threshold, the replication rate of the more malignant subpopulation, $x_{2}$ is reduced to a point where it exhibits no competitive advantage anymore. According to the clonal expansion concept, this will soon lead to the extinction of these highly aggressive $x_{2}$-cells from the overall cancer cell population, which in turn induces much slower, i.e. slow tumor growth dynamics.

The phase transition between these two growth regimes is both sharp and robust. A recent analysis (Solé, 2003) using a bit string description of the sequences confirms the validity of the mean field model. The clear distinction between these phase spaces demonstrates that there is a growth advantage for tumor cells operating with a limited amount of genetic instability. However, if the level of genetic instability exceeds a certain threshold, the deleterious effects of this instability outweigh by far its beneficial effects of being able to survive a variety of environmental stresses (Li et al., 2001). A tumor cell population, in which instability exceeds this threshold rapidly looses fitness and evidently breaks down. A similar concept has already been suggested by Cahill et al. (1999) on the basis of insights from studies on bacteria.

Our findings yield several novel insights into tumor progression. Uninhibited cell proliferation is a hallmark of malignancy and recent reports argue that genetic instability confers a selection advantage for tumors in order to escape recognition by the immune system (for a review see Khong and Restifo, 2002). Environmental stresses such as conferred by the immune system itself (Pettit et al., 2000) apply selection pressure, hence potentially triggering an increase in the tumor's genetic instability and thus ultimately enabling the tumor to evade the very same (immune) system. Taken together, our results argue that malignant tumors may in fact progress towards both, maximum proliferation rate and maximum genetic instability. If so, the target the tumor optimization process is of course in the malignant domain of phase space, yet close to the phase transition (Figs. 3 and 4).

The implications for the development of novel treatment strategies seem grim at first. Many treatment approaches such as adjuvant immunotherapy will inevitably select for traits that facilitate immune evasion, thus increase the tumor cell population harboring genetic instability and thereby ensure further growth. Conversely, selectively inducing more genetic instability within the tumor cell populations may have some therapeutic potential. For example, by specifically inhibiting the DNA repair enzyme alkyltransferase with 
O6-benzylguanine Rhines et al. (2000) were able to potentiate the activity of the anti-proliferative agent BCNU. More work will therefore be necessary to determine the impact of different treatment regimens on the phase transition.

In its underlying conceptual framework, the model combines the existence of a mutator-phenotype population, responsible for the marked increase in genetic instability, with a selection-driven, clonal expansion process. As such, our model incorporates both previously thought to be mutually exclusive concepts and suggests that this combination may function as a driving force behind the progression of malignant tumors. In this context, treatment would exploit an intrinsic feature of unstable tumors that is actually an Achilles heel for cancer. The recent success in the removal of RNA viruses from cell cultures by taking advantage of the error threshold suggests that genetic destabilization could be a promising strategy also in anticancer therapy.

\section{Acknowledgements}

We thank Isabel González-Garcia (who suggested interesting links between RNA viruses and cancer) and to Josep Costa for useful discussions. This work has been supported by grants MCYT BFM 2001-2154 and by the Santa Fe Institute (RVS) and by the HarvardMIT (HST) Athinoula A. Martinos Center for Biomedical Imaging, the Department of Radiology and the Molecular Neuro-Oncology Laboratory at Massachusetts General Hospital (TSD).

\section{References}

Bardelli, A., Cahill, D.P., Lederer, G., Speicher, M.R., Kinzler, K.W., Vogelstein, B., Lengauer, C., 2001. Carcinogen-specific induction of genetic instability. Proc. Natl. Acad. Sci. USA 98, 5770-5775.

Cahill, D.P., Kinzler, K.W., Vogelstein, B., Lengauer, C., 1999. Genetic instability and darwinian selection in tumors. Trends Genet. 15, M57-M61.

Chow, M., Rubin, H., 2000. Clonal selection versus genetic instability as the driving force in neoplastic transformation. Cancer Res. 60, 6510-6518.

Coffin, J.M., 1995. HIV population dynamics in vivo: implications for genetic variation, pathogenesis and therapy. Science 267, 483-489.

Cottry, S., Cameron, C.E., Andino, R., 2001. RNA virus error catastrophe: direct molecular test by using ribavirin. Proc. Natl. Acad. Sci. USA 98, 6895-6900.

Domingo, E., Holland, J.J. (Eds.), 1994. Mutation rates and rapid evolution of RNA viruses. In: Morse, S. (Ed.), The Evolutionary Biology of RNA Viruses. Raven Press, New York, pp. 161-183.

Domingo, E., Holland, J.J., Biebricher, C., Eigen, M., 1995. Quasispecies: the concept and the word. In: Gibbs, A., Calisher, C., Garcia-Arenal, F. (Eds.), Molecular Evolution of the Viruses. Cambridge University Press, Cambridge.

Eigen, M., 1971. Self-organization of matter and the evolution of biological macromolecules. Naturwiss. 58, 465-523.
Eigen, M., McCaskill, J., Schuster, P., 1987. The molecular quasispecies. Adv. Chem. Phys. 75, 149-263.

Eigen, M., McCaskill, Schuster, P., 1988. Molecular quasi-species. J. Phys. Chem. 92, 6881-6891.

Fearon, E.R., Vogelstein, B., 1990. A genetic model for colorectal tumorigenesis. Cell 61, 759-767.

Fishel, R., 2001. The selection for mismatch repair defects in hereditary nonpolyposis colorectal cancer: revising the mutator hypothesis. Cancer Res. 61, 7369-7374.

Frank, S.A., Iwasa, Y., Nowak, M.A., 2003. Patterns of cell division and the risk of cancer. Genetics 163 (4), 1527-1532.

Gatenby, R.A., 1995. Models of tumor-host interactions as competing populations. J. Theor. Biol. 176, 447-455.

Gatenby, R.A., 1996. Application of competition theory to tumour growth: implications for tumour biology and treatment. Eur. J. Cancer 32A, 722-726.

Gatenby, R.A., Frieden, B.R., 2002. Application of information theory and extreme physical information to carcinogenesis. Cancer Res. 62, 3675-3684.

Holland, J.J., Domingo, E., de la Torre, J.C., Steinhauer, D.A., 1999. Mutation frequencies at defined single codon sites in vesicular stomatitis virus and poliovirus can be increased only slightly by chemical mutagenesis. J. Virol. 64, 3960-3962.

Jackson, J., Loeb, L.A., 1998. The mutation rate and cancer. Genetics 148, 1483-1490.

Kamp, C., Bornholdt, S., 2002. Co-evolution of quasispecies: B-cell mutation rates maximize viral error catastrophes. Phys. Rev. Lett. $88,068104$.

Kamp, C., Wilke, C.O., Adami, S., Bornholdt, S., 2003. Viral evolution under the pressure of an adaptive immune systemoptimal mutation rates for viral escape. Complexity 8 (2), 28-32.

Khong, H.T., Restifo, N.P., 2002. Natural selection of tumor variants in the generation of "tumor escape" phenotypes. Nat. Immunol. 3, 999-1005.

Lengauer, C., Kinzler, K.W., Vogelstein, B., 1998. Genetic instabilities in human cancers. Nature 396, 643-649.

Loeb, L.A., 1991. Mutator phenotype may be required for multistage carcinogenesis. Cancer Res. 51, 3075-3079.

Loeb, L.A., 1994. Microsatellite instability: marker of a mutator phenotype in cancer. Cancer Res. 54, 5059-5063.

Loeb, L.A., Essigmann, J.M., Kazazi, F., Zhang, J., Rose, K.D., Mullins, J.I., 1999. Lethal mutagenesis of HIV with mutagenic nucleoside analogs. Proc. Natl. Acad. Sci. USA 96, 1492-1497.

Loeb, L.A., Loeb, K.R., Anderson, J.P., 2003. Multiple mutations and cancer. Proc. Natl. Acad. Sci. USA 100, 776-781.

Li, C.-Y., Little, J.B., Hu, K., Zhang, W., Zhang, L., Dewhirst, M.W., Huang, Q., 2001. Persistent genetic instability in cancer cells induced by non-DNA-damaging stress exposures. Cancer Res. 61, 428-432.

Miller, B.E., Miller, F.R., Heppner, G.H., 1981. Interactions between tumor subpopulations affecting their sensitivity to the antineoplastic agents cyclophosphamide and methotrexate. Cancer Res. 41, 4378-4381.

Miller, B.E., Miller, F.R., Wilburn, D.J., Heppner, G.H., 1987. Analysis of tumour cell composition in tumours composed of paired mixtures of mammary tumour cell lines. Br. J. Cancer 56, 561-569.

Novell, P.C., 1976. The clonal evolution of tumor cell populations. Science 194, 23-28

Nowak, M., May, R.M., 2000. Virus Dynamics. Oxford University Press, Oxford.

Pastor-Satorras, R., Solé, R.V., 2001. Field theory of a reactiondiffusion model of quasispecies dynamics. Phys. Rev. E 64, 051909.

Pettit, S.J., Seymour, K., O'Flaherty, E., Kirby, J.A., 2000. Immune selection in neoplasia: towards a microevolutionary model of cancer development. Br. J. Cancer 82, 1900-1906. 
Plotkin, J.B., Nowak, M.A., 2002. The different effects of apoptosis and DNA repair on tumorigenesis. J. Theor. Biol. 214, 453-467.

Poste, G., Doll, J., Fidler, I.J., 1981. Interactions among clonal subpopulations affect stability of the metastatic phenotype in polyclonal populations of B16 melanoma cells. Proc. Natl. Acad. Sci. USA 78, 6226-6230.

Rhines, L.D., Sampath, P., Dolan, M.E., Tyler, B.M., Brem, H., Weingart, J., 2000. O6-benzylguanine potentiates the antitumor effect of locally delivered carmustine against an intracranial rat glioma. Cancer Res. 60, 6307-6310.

Rosenberg, S.A., 2001. Progress in human tumour immunology and immunotherapy. Nature 411, 380-384.

Schuster, P., 1994. How do RNA molecules and viruses explore their worlds? In: Cowan, G.A., Pines, D., Meltzer, D. (Eds.), Complex- ity: Metaphors, Models and Reality. Addison-Wesley, Reading, MA, pp. 383-418.

Solé, R.V., 2003. Phase transitions in unstable cancer cell populations. Eur. Phys. J. B 35, 117-124.

Solé, R.V., Goodwin, B.C., 2001. Signs of Life: How Complexity Pervades Biology. Basic Books, Perseus, NY.

Solé, R.V., Manrubia, S., Luque, B., Delgado, J., Bascompte, J., 1996. Phase transitions and complex systems. Complexity 1 (4), 13-26.

Swetina, J., Schuster, P., 1982. Self-replication with errors. A model for polynucleotide replication. Biophys. Chem. 16, 329-345.

Tomlinson, I.P.M., Novelli, M.R., Bodmer, W.F., 1996. The mutation rate and cancer. Proc. Natl. Acad. Sci. USA 93, 14800-14803.

Wodarz, D., Krakauer, D., 2001. Genetic instability and the evolution of angiogenic tumor cell lines (review). Oncology Rep. 8, 1195-1201. 